\title{
Models for Type Ia Supernovae and Evolutionary Effects with Redshift
}

\author{
P. Höflich \\ Department of Astronomy, University of Texas, Austin, TX 78681, USA
}

\begin{abstract}
Based on detailed models for the explosions, light curves and NLTEspectra, evolutionary effects of Type Ia Supernovae (SNe Ia) with redshift have been studied to evaluate their size on cosmological time scales, how the effects can be recognized and how one may be able to correct for them.

In the first part, we summarize the current status of scenarios for Type Ia Supernovae, including the explosion of a Chandrasekhar mass white dwarf $\left(M_{C h}\right.$-WD), the merging scenario and the helium-triggered explosions of low-mass WDs. We show that delayed detonation models can account for the majority of observations of spectra and light curves. IR observations are a new and powerful tools to constrain explosion models and details of the flame propagation in the WD. A strong Mg II line at $1.05 \mu \mathrm{m}$ shows that nuclear burning takes place at the outer, low density layers. This requires a transition from the deflagration to the detonation regime of the nuclear burning front, or a very fast deflagration close to the speed of sound. We put the models into context with the empirical brightness decline relation which is widely applied to use $\mathrm{SNe}$ Ia as yardsticks on cosmological distance scales. This relation can be well understood in the framework of $M_{C h}$-WDs as a consequence of the opacity effects in combination with the amount of ${ }^{56} \mathrm{Ni}$ which determines the brightness. However, the amount of ${ }^{56} \mathrm{Ni}$ actually produced depends on a combination of free parameters such as central density and the chemical composition of the WD, and the propagation of the burning front. We get a spread of $\approx 0.4^{m}$ around the mean relation which is larger than currently favored by observations $\left(\approx 0.12^{m},[40]\right)$ which may hint of an underlying coupling of the progenitor, the accretion rates and the propagation of the burning front.

In a second part, we investigate the possible evolutionary effects in SNe Ia both with respect to changes in the sample of SNe Ia and individual variations, and how they can be identified. We find that evolution may produce an offset in the brightness decline relation is restricted to a few tenth of a magnitude. The effects reveal themself by changes in the $\mathrm{U}$ and UV fluxes, and in a change in the maximum brightness/decline relation by $\Delta M \approx 0.1 \times \Delta t$ where $\Delta t$ is the difference between local and distant SN-samples. According to new data [1], $\Delta t \leq 1 d$ and, thus, evolution is unlikely to eliminate a need for $\Lambda$.
\end{abstract}

\section{INTRODUCTION}

Two of the important developments in observational SN-research in the last few years were to establish the long-suspected correlation between the peak brightness 
of SNe Ia and their rate of decline by means of modern CCD photometry [38], and the exact distance calibrations provided by an HST Key Project (e.g. [41]). Both allowed a empirical determinations of $H_{o}$ with unprecedented accuracy [11]. Independent from these calibrations and empirical relations, $H_{o}$ has been determined by comparisons of detailed theoretical models for light curves and spectra with observations $[27,16,35]$. All determinations of the Hubble constant are in good agreement with one another. Recently, the routine successful detection of SNe at large redshifts, z $[39,40]$, has provided an exciting new tool to probe cosmology. This work has provided results that are consistent with a low matter density in the Universe and, most intriguing of all, yielded hints for a positive cosmological constant. The cosmological results rely on empirical brightness-decline relations which are calibrated locally. One of the main concerns are systematic, evolutionary effects in the properties of SNe Ia. Both from theory and observations, there are some hints for the presents of evolution.

Observational evidence include the finding of Branch et al. [5] who have shown that the mean peak brightness is dimmer in ellipticals than in spiral galaxies. Wang, Höflich \& Wheeler [47] found that the peak brightness in the outer region of spirals is similar to those found in ellipticals, but that in the central region both intrinsically brighter and dimmer SNe Ia occur. This implies that the progenitor populations are more inhomogeneous in the inner parts of spirals which contain both young and old progenitors.

From theory, time evolution is expected to produce the following main effects: (a) a lower metallicity will decrease the time scale for stellar evolution of individual stars by about $20 \%$ from Pop I to Pop II stars [42] and, consequently, the progenitor population which contributes to the SNe Ia rate at any given time. The stellar radius also shrinks. This will influence the statistics of systems with mass overflow; (b) early on, we expect that systems with shorter life time will dominate the sample whereas, today, old system are contributing which may have not occurred early on. In addition, some scenarios with a life time comparable to the age of the univers such as two merging WDs may be absent a few Gyrs ago, but they may contribute today. (c) The initial metallicity will determine the electron to nucleon fraction of the outer layers and hence affects the products of nuclear burning; (d) Systems with a shorter life time may dominate early on and, consequently, the typical C/O ratio of the central region of the WD will be reduced; (e) The properties of the interstellar medium may change; (f) The influence of $\mathrm{Z}$ on the structure of WDs may change, but this effect has remains very small (e.g. $2 \%$ when comparing solar with 0.01 solar, [19]) (g) The distribution of $\mathrm{C}$ and $\mathrm{O}$ will depend on $\mathrm{Z}$ as it influences the 'normal' stellar evolution and the properties of the $\mathrm{C} / \mathrm{O}$ core [42] (h) The metallicity will effect nuclear burning during the accretion phase of the progenitor [34].

Based on theoretical models described in §III, we want to study the possible effects of the change of the progenitor and its metallicity on the light curves and spectra of SNe Ia. Note that most of the results have been obtained in serveral collaborations over the years (see acknowledgments). 


\section{NUMERICAL TOOLS}

Most of the results discussed in the following sections are based on our calculations which are consistent with the explosion, light curves and spectra. In some cases, the stellar evolution and the accretion on the WD is treated in detail.

Stellar Evolution: The stellar models have been calculated using the evolutionary code Franec (e.g. [43,6,8]) or are provided by Nomoto's group [46]. Subsequently, the evolution of the $\mathrm{C} / \mathrm{O}$ core is followed up by accreting $\mathrm{H} / \mathrm{He}$ rich material at a given accretion rate on the core by solving the standard equations for stellar evolution using a Henyey scheme. Nomoto's equation of state is used [32]. Crystallization is neglected. For the energy transport, conduction [22], convection in the mixing length theory, and radiation is taken into account. Radiative opacities for free-free and bound free transitions are approximated in Kramer's approximation and by free electrons. A nuclear network of 35 species up to ${ }^{24} \mathrm{Mg}$ is used.

Hydrodynamics: The explosions are calculated using a one-dimensional radiation-hydro code, including nuclear networks ( [16] and references therein). This code solves the hydrodynamical equations explicitly by the piecewise parabolic method [7] and includes the solution of the frequency averaged radiation transport implicitly via moment equations, expansion opacities (see below), and a detailed equation of state. Nuclear burning is taken into account using a network which has been tested in many explosive environments (see [44] and references therein).

Light Curves: Based on the explosion models, the subsequent expansion and bolometric as well as monochromatic light curves are calculated using a scheme recently developed, tested and widely applied to SN Ia (e.g. $[13,19])$. The code used in this phase is similar to that described above, but nuclear burning is neglected and $\gamma$ ray transport is included via a Monte Carlo scheme. In order to allow for a more consistent treatment of scattering, we solve both the (two lowest) time-dependent, frequency averaged radiation moment equations for the radiation energy and the radiation flux, and a total energy equation. At each time step, we then use $T(r)$ to determine the Eddington factors and mean opacities by solving the frequency-dependent radiation transport equation in the comoving frame and integrate to obtain the frequency-averaged quantities. About 1000 frequencies (in one 100 frequency groups) and about 500 to 1000 depth points are used. The averaged opacities have been calculated under the assumption of local thermodynamic equilibrium. Both the monochromatic and mean opacities are calculated using the Sobolev approximation. The scattering, photon redistribution and thermalization terms used in the light curve opacity calculation are calibrated with NLTE calculations using the formalism of the equivalent-two-level approach [14].

Spectral Calculations: Our non-LTE code (e.g. [14,19]) solves the relativistic radiation transport equations in comoving frame. The energetics of the SN are calculated. The evolution of the spectrum is not subject to any tuning or free parameters. The spectra are computed for various epochs using the chemical, density and luminosity structure and $\gamma$-ray deposition resulting from the light curve code providing a tight coupling between the explosion model and the radiative 
transfer. The effects of instantaneous energy deposition by $\gamma$-rays, the stored energy (in the thermal bath and in ionization) and the energy loss due to the adiabatic expansion are taken into account. The radiation transport equations are solved consistently with the statistical equations and ionization due to $\gamma$ radiation for the most important elements (C, O, Ne, Na, Mg, Si, S, Ca, Fe, Co, Ni). Besides 40,000 lines treated in full non-LTE, $\approx 10^{6}$ additional lines are included assuming LTElevel populations and an equivalent-two-level approach for the source functions.

\section{MODELS FOR THERMONUCLEAR SUPERNOVAE}

There is general agreement that SNIa result from the thermonuclear explosion of a degenerate white dwarf (HF60). Within this general picture, three classes of models have been considered: (1) An explosion of a CO-WD, with mass close to the Chandrasekhar mass, which accretes mass through Roche-lobe overflow from an evolved companion star [30]. The explosion is then triggered by compressional heating near the WD center. (2) An explosion of a rotating configuration formed from the merging of two low-mass WDs, caused by the loss of angular momentum due to gravitational radiation $[48,21,37]$. The explosion may also be triggered near the center by the compression. (3) Explosion of a low mass CO-WD triggered by the detonation of a helium layer [31,51]. Only the first two models appear to be viable. The third, the sub-Chandrasechar WD model, has been ruled out on the basis of predicted light curves and spectra $[18,36]$.

From the theoretical standpoint, one of the key questions is how the flame propagates through the WD. Several burning models have been proposed in the past, including detonation [2,12], deflagration [23,29], and the delayed detonation model (DD) $[26,50,52]$. The DD-model assumes that burning starts as a subsonic deflagration with a certain speed $S_{\text {def }}<a_{s}$, where $a_{s}$ is the sound speed, and then undergoes a transition to a supersonic detonation. The detonation speed follows directly from the standard Hugoniot relations. However, due to the one-dimensional nature of current model, the speed of the subsonic deflagration and the moment of the transition to a detonation are free parameters, or calibrated by $3-\mathrm{D}$ calculations. The moment of deflagration-to-detonation transition (DDT) is conveniently parameterized by introducing the transition density, $\rho_{\mathrm{tr}}$, at which DDT happens. Recently, significant progress has been made toward a better understanding of the propagation of nuclear burning fronts during the deflagration phase. The models provide good results during the phase when the Ryleigh-Taylor instabilites dominate but the results depend sensitively on the assumed sub-grid models at later phase of the flame propagation $[24,25,28]$, and the DDT-transition is not understood quanitatively. Therefore, further constraints must come from the observations.

What we observe as a SN is not the explosion itself, but the light emitted from a rapidly expanding ejecta produced by the explosion. As the photosphere recedes, deeper layers of the ejecta become visible. A detailed analysis of multi-band light curves and spectra gives us the opportunity to determine the density, velocity and 
composition structure of the ejecta, and to constrain the physical model of the explosion. The major results of one-dimensional modeling and comparison with observations are summarized below.
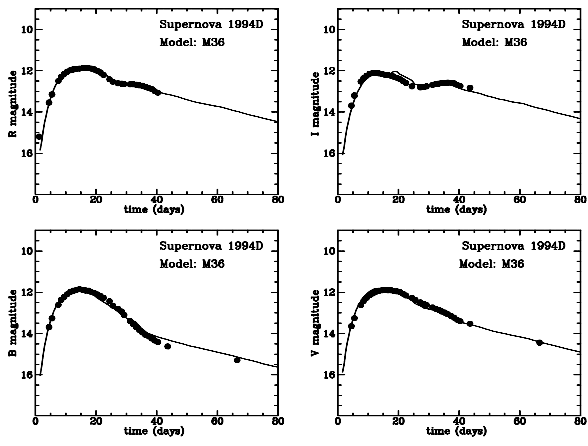

FIGURE 1. Observed LCs of SN 1994D in comparison with the theoretical LCs of a typical delayed detonation model, and the corresponding synthetic spectrum around bolometric maximum in comparison to the observations of SN1994D at Mar. 16th (from Höflich 1995).
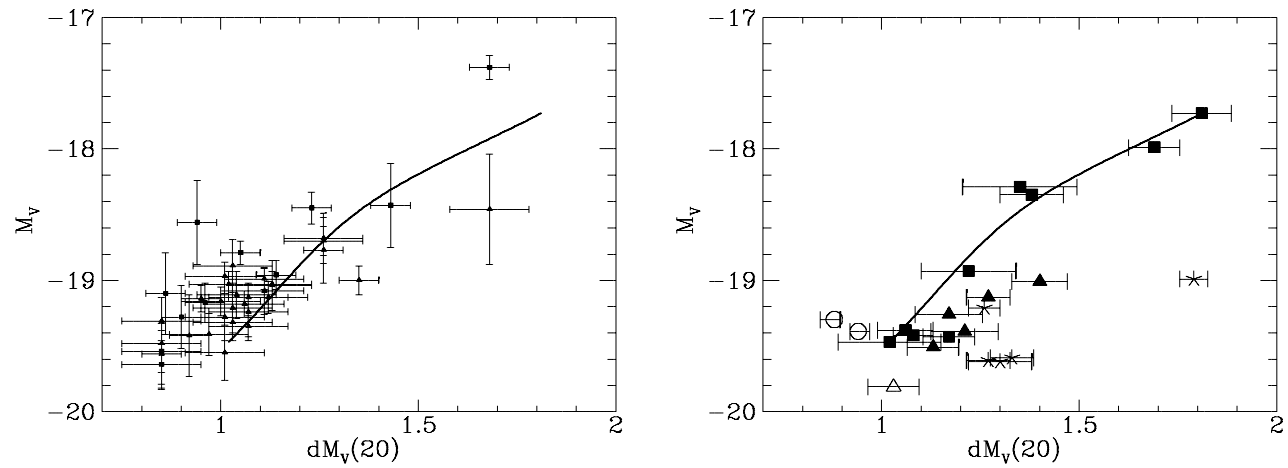

FIGURE 2. (Left panel) Observed light curve maximum brightness - decline rate relation. $\mathrm{M}_{V}$ is presented as a function of the decline from maximum at 20 days. (Right panel) The predicted relation for an array of models of SNe Ia representing delayed detonations (open triangles), pulsating delayed detonations (filled circles), merging models (open circles) and helium detonations (asterisks). For both the delayed detonation and merger scenarios models are only considered if they allow for a representation of some of the observed SNe Ia (Höflich et al. 1996).

The best current explosion model, the delayed detonation and pulsating delayed detonation explosion models of Chandrasekhar mass carbon-oxygen WDs can account for the spectral and light curve evolution of both "normally bright" and subluminous SNIa in the optical and IR (see Fig. 1, [14-16,49]. Within this framework, we can understand for often used brightness decline relation. For a plausible range in the transition density, $\rho_{\mathrm{tr}} \simeq(1.5-2.5) \times 10^{7} \mathrm{~g} \mathrm{~cm}^{-3}[16]$, sufficient thermonuclear energy is generated by burning nearly the entire WD to provide the observed expansion velocities, a small spread in the explosion energy [14]. The amount of ${ }^{56} \mathrm{Ni}$ varies between $\simeq 0.1-0.7 \mathrm{M}_{\odot}$. The variation of $M_{\mathrm{Ni}}$ gives a range in maximum brightness that matches the observations (Fig. 2). The models with less nickel are not only dimmer, but are cooler and have lower opacity, giving 
them redder, more steeply declining light curves, in agreement with the observations [13,17]. The amount of ${ }^{56} \mathrm{Ni}$ depends primarily on $\rho_{t r}$, and to a much lesser extent on the assumed value of deflagration speed, initial central density of the exploding star, and the initial chemical composition (ratio of carbon to oxygen). This is the basis of why, to first approximation, SNIa appear to be a one-parameter family. Nonetheless, variations of the other parameters also lead to some variations of the predicted properties of SNIa, which indicate that the assumption of a oneparameter family is not strictly valid. We get a spread around the mean relation of $\approx 0.4^{m}$ which is consistent with the spread based on the CTIO data published by Hamuy et al. [11] but larger than suggested by recent observations $\left(\approx 0.12^{m}[40]\right)$. This narrow a spread cannot be understood in the context of current models but it may hint of an underlying coupling of the progenitor, the accretion rates and the propagation of the burning front.
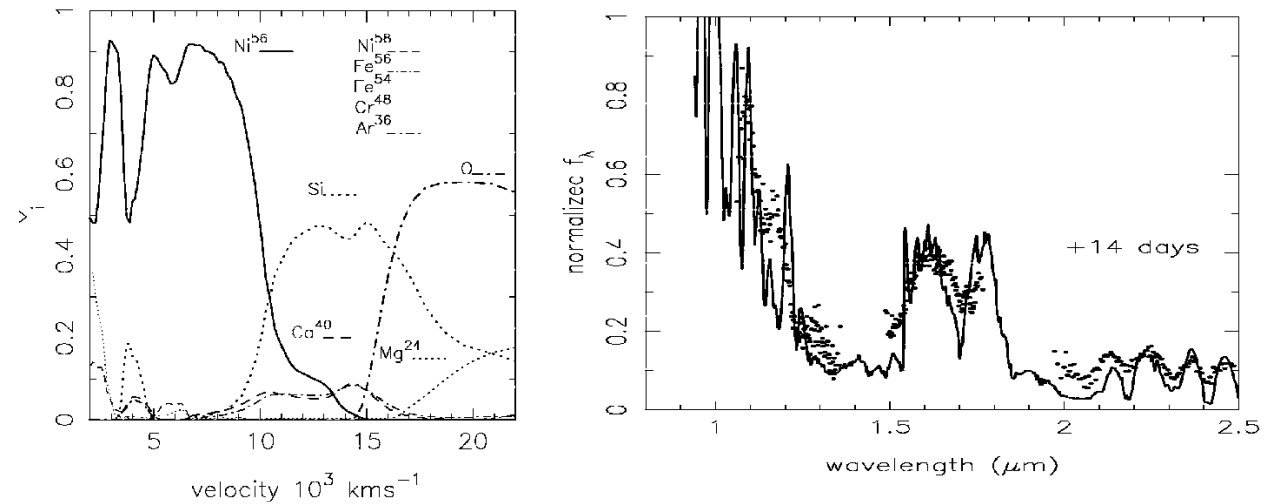

FIGURE 3. Final chemical structure of a delayed detonation model as calculated in 1-D in comparison and the theoretical IR-spectrum in the IR at about 2 weeks after maximum light in comparison with the observed spectrum of $1976 \mathrm{~g}$ at about +10 days by Bowers et al. (1997). The emission feature between 1.5 and $1.8 \mu \mathrm{m}$ is produced by a large number of $\mathrm{Fe}$, Co and Ni lines. The ${ }^{56} \mathrm{Ni}$ layers are optically thick at these wavelengths. It appears as soon as the 'photosphere' at adjoining wavelengths receds to velocities smaller than the outer edge of the ${ }^{56} \mathrm{Ni}$ layers (from Wheeler, Höflich, Harkness and Spyromillo 1998).

During the last 2 to 3 years, near IR spectra became available and provided a new insights. Firstly, the comparison with observed IR spectra with model predictions was an important test for the models since there was no a priori guarantee that a model that matched optical light curves would produce satisfactory IR spectra (Fig. 3, [49] ). The broad feature between 1.5 and $1.8 \mu \mathrm{m}$ is produced by a large number of $\mathrm{Fe}$, Co and $\mathrm{Ni}$ lines and indicates the size of the ${ }^{56} \mathrm{Ni}$ rich regions. A very strong Mg II line at about $1.05 \mu \mathrm{m}$ at early times can be understood as a natural consequence of burning in DD-models. Because $\mathrm{Mg}$ is produced in the region of explosive carbon burning, the Doppler shift provides a unique tool to determine the transition zone from explosive carbon to oxygen burning. Its high, minimum velocity clearly demonstrates the need for a DDT transition or a deflagration speed well in excess of those of the deflagration model W7 [33], and it rules out strong 
mixing as a possible explanation for the presence of high velocity Si frequently seen in 'normal' bright SNe Ia [3,9].

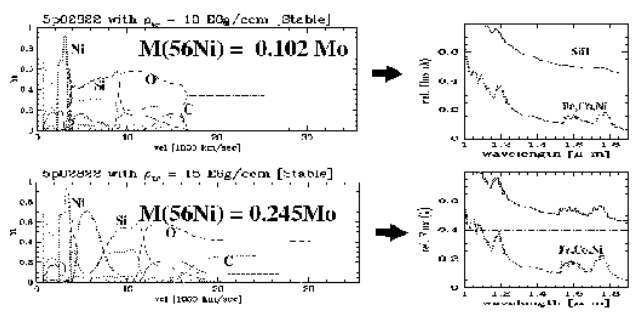

FIGURE 4. Final chemical structure of two strongly subluminous delayed detonation model and the corresponding, theoretical IR-spectrum at 1 and 2 weeks after maximum light. In the strongly subluminous SN1999bu, the 1.5 to $1.8 \mu \mathrm{m}$ feature was not observed until about 2 weeks after maximum light. To be consistent with this observation, the abundance ${ }^{56} \mathrm{Ni}$ must be less than $\approx 10 \%$ at layers with expansion velocities in excess of $\approx 5000 \mathrm{~km} / \mathrm{sec}$ Gerardy et al. (2000).

Recently, IR for the very subluminous SN1999bu by Gerardy et al. [10] shows no $\mathrm{Ni}$ at layers with expansion velocities larger than $\approx 5000 \mathrm{~km} / \mathrm{sec}$. Progress in combustion in SNeIa predicted (e.g. [24]) that, during the deflagration phase, some Ni bubbles may rise to about half of the WD-radius corresponding to $0.4 M_{\odot}$ which may be inconsistent with results of the 1-D spectra (Fig.4) because, in subluminous SNe Ia, most of the ${ }^{56} \mathrm{Ni}$ is produced during the deflagration phase. The solution to this problem may prove to be the Rosetta stone for understanding the influence of the preconditioning of the WD, i.e. the progenitor star, its rotation etc., and of propagation of burning fronts. Eventually, it may help to answer the question whether a SNIe Ia is subluminous because its special initial conditions influence the burning front, or whether current models for the deflagration need to be modified.

\section{EVOLUTIONARY EFFECTS}

A change of the main sequence mass of the typical progenitor will change the size the region of central He burning during the stellar evolution and, consequently, the size and $\mathrm{C} / \mathrm{O}$ ratio in the WD which, eventually, may explode as a SNe Ia. The initial metallicity $\mathrm{Z}$ of the $\mathrm{WD}$ is inherented from the molecular cloud from which the star has been formed. The influence of $\mathrm{Z}$ and $\mathrm{C} / \mathrm{O}$ ratio on light curves and spectra has been studied for the example of a set of DD-models with the basic properties as follows: central density of the WD, $\rho_{c}=2.6 \times 10^{9} \mathrm{~g} / \mathrm{cm}^{-3}$, $v_{\text {burn }}=\alpha * v_{\text {sound }}$ with $\alpha=0.03$ during the deflagration phase and a transition to detonation at $\rho_{t r}=2.7 \times 10^{7}$. The quantities of Table 1 in columns 2 to 5 and 6 
TABLE 1. Basic parameters for the delayed detonation models.

\begin{tabular}{llllllllll} 
Model & $\mathrm{C} / \mathrm{O}$ & $R_{Z}$ & $E_{k i n}$ & $M_{N i}$ & Model & $\mathrm{C} / \mathrm{O}$ & $R_{Z}$ & $E_{k i n}$ & $M_{N i}$ \\
\hline DD21c & $1 / 1$ & $1 / 1$ & 1.32 & 0.69 & DD25c & $1 / 1$ & $3 / 1$ & 1.32 & 0.69 \\
DD23c & $2 / 3$ & $1 / 1$ & 1.18 & 0.59 & DD26c & $1 / 1$ & $1 / 10$ & 1.32 & 0.73 \\
DD24c & $1 / 1$ & $1 / 3$ & 1.32 & 0.70 & DD27c & $1 / 1$ & $10 / 1$ & 1.32 & 0.69 \\
\hline
\end{tabular}

to 9 are: $\mathrm{C} / \mathrm{O}$ ratio; $R_{Z}$ the $\mathrm{Z}$ relative to solar by mass; $E_{k i n}$ kinetic energy (in $10^{51} \mathrm{erg}$ ); $M_{N i}$ mass of ${ }^{56} \mathrm{Ni}$ (in solar units). The parameters are close to those which reproduce both the spectra and light curves reasonably well $[33,14]$.
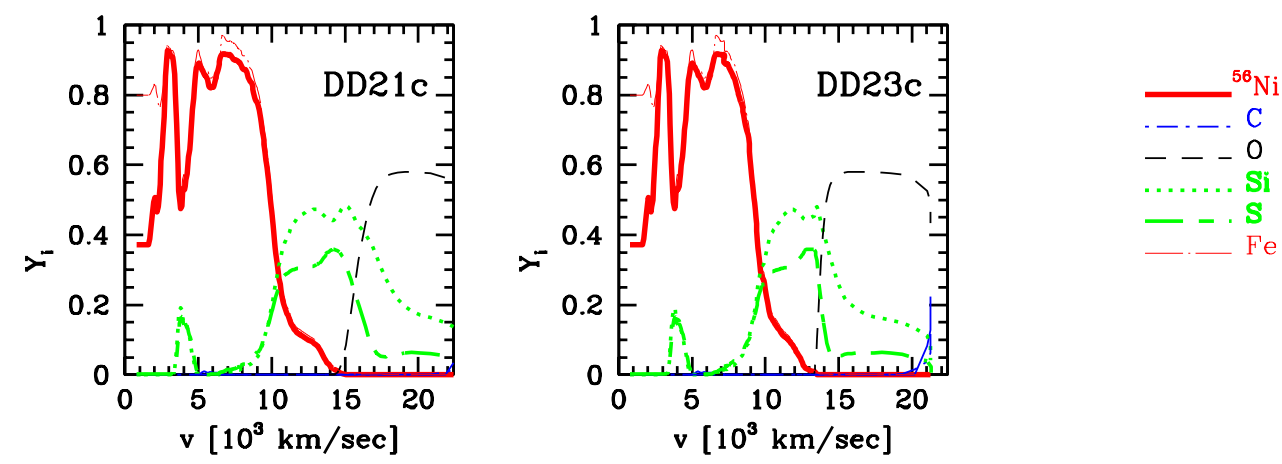

FIGURE 5. Abundances as a function of the final expansion velocity for the delayed detonation models DD21c and DD23c. Both the initial ${ }^{56} \mathrm{Ni}$ and the final Fe profiles are shown.

Direct influence of C/O: As the C/O ratio of the WD is decreased from 1 to 2/3, the explosion energy and the ${ }^{56} \mathrm{Ni}$ production are reduced and the Si-rich layers are more confined in velocity space (Fig. 5). A reduction of $\mathrm{C} / \mathrm{O}$ by about $60 \%$ gives slower rise times by about 3 days and an increased luminosity at maximum light, a somewhat faster post-maximum decline and a larger ratio between maximum light and the ${ }^{56} \mathrm{Co}$ tail ( [19], see also Fig.8). The slight increase in luminosity at maximum light is caused by the smaller expansion rate. Consequently, less energy stored early on is wasted in expansion work, but contributes to the luminosity. The smaller ${ }^{56} \mathrm{Ni}$ production causes the reduction of the luminosity later on.

A reduction of the $\mathrm{C} / \mathrm{O}$ ratio has a similar effect on the colors, light curve shapes and element distribution as a reduction in the deflagration to detonation transition density but, for the same light curve shape, the absolute brightness is larger for smaller $\mathrm{C} / \mathrm{O}$. Moreover, the kinetic energy is reduced by about $10 \%$ (Table 1) and, consequently, the expansion velocity derived by the Doppler shift in the spectra becomes smaller by about $5 \%$. An independent determination of the initial $\mathrm{C} / \mathrm{O}$ ratio and the transition density is possible for local SN if detailed analyses of both the spectra and light curves are performed simultaneously.

Direct influence of the metallicity: To test the influence of the metallicity for nuclei beyond $\mathrm{Ca}$, we have constructed models with parameters identical to DD21c but with initial metallicities between 0.1 and 10 times solar (Table 1). The energy release, the density and velocity structure are virtually identical to that of DD21c 
and, consequently, the bolometric (and also monochromatic) optical light curves are rather insensitive. The main influence of $\mathrm{Z}$ is a slight increase of the ${ }^{56} \mathrm{Ni}$ mass with decreasing $\mathrm{Z}$ due to a higher $Y_{e}$. The reason is that $\mathrm{Z}$ mainly effects the initial $\mathrm{CNO}$ abundances of a star. These are converted during the pre-explosion stellar evolution to ${ }^{14} \mathrm{~N}$ in H-burning and via ${ }^{14} \mathrm{~N}(\alpha, \gamma){ }^{18} \mathrm{~F}\left(\beta^{+}\right){ }^{18} \mathrm{O}(\alpha, \gamma){ }^{22} \mathrm{Ne}$ to nuclei with $\mathrm{N}=\mathrm{Z}+2$ in He-burning. The result is that increasing $\mathrm{Z}$ yields a smaller proton to nucleon ratio $Y_{e}$ throughout the pre-explosive WD. Higher $\mathrm{Z}$ and smaller $Y_{e}$ lead to the production of more neutron-rich Fe group nuclei and less ${ }^{56} \mathrm{Ni}$ (Fig. 6). For lower $\mathrm{Z}$ and, thus, higher $Y_{e}$, some additional ${ }^{56} \mathrm{Ni}$ is produced at the expense of ${ }^{54} \mathrm{Fe}$ and ${ }^{58} \mathrm{Ni}[45]$. The temperature in the inner layers is sufficiently high during the explosion that electron capture determines $Y_{e}$. In those layers, $\mathrm{Z}$ has no influence on the final burning product. The main differences due to changes in $\mathrm{Z}$ are in regions with expansion velocities in excess of $\approx 12000 \mathrm{~km} / \mathrm{sec}$. Most remarkable is the change in the ${ }^{54} \mathrm{Fe}$ production which is the dominant contributor to the abundance of iron group elements at these velocities since little cobalt has yet decayed near maximum light (Fig. 6).
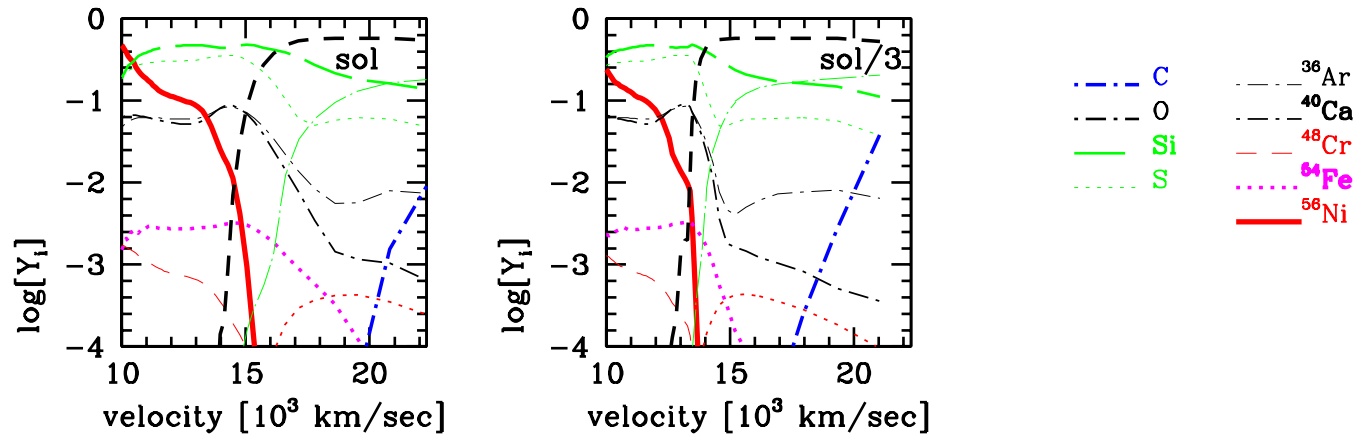

FIGURE 6. Abundances of different isotopes as a function of the expansion velocity for DD21c with initial abundances of solar and solar $/ 3$.

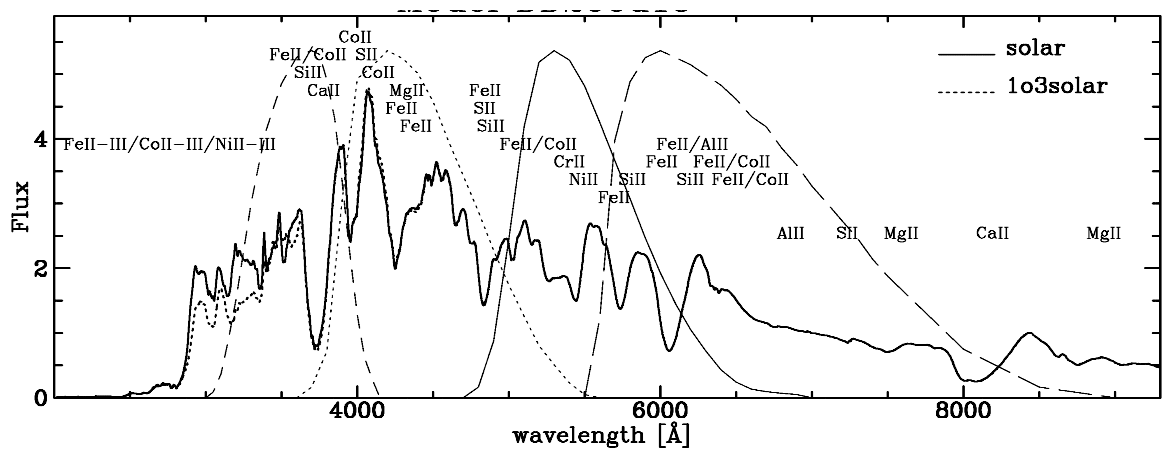

FIGURE 7. Comparison of synthetic NLTE spectra at maximum light for Z solar (DD21c) and $1 / 3$ solar (DD24c). The standard Johnson filter functions for UBV, and R are also shown.

The initial WD composition has been found to have rather small effects on the overall LCs. The ${ }^{56} \mathrm{Ni}$ production and hence the bolometric and monochromatic optical and IR light curves differ only by a few hundredths of a magnitude. This change is almost entirely due to the small change in the ${ }^{56} \mathrm{Ni}$ production and hardly 
due to a change in the opacities because the diffusion time scales are governed by the deeper layers where burning is complete. However, the short wavelength part of the spectrum $(\lambda \leq 4000 \AA)$ at maximum light is affected by a change in $\mathrm{Z}$ (Fig.7). This provides a direct test for $\mathrm{Z}$ of local SNe and, thus, may give a powerful tool to unravel the nature (and lifetime) of SNe Ia progenitors.

By 2 to 3 weeks after maximum, the spectra are completely insensitive to the initial $\mathrm{Z}$ because the spectrum is formed in even deeper layers where none of the important abundances are effected by Z. Thus, for two similar bright SNe with similar expansion velocities, a comparison between the spectral evolution can provide a method to determine the difference in Z, or it may be used to detect evolutionary effects for distant SNe Ia if high quality spectra are available.

Influence of the Stellar Evolution on the WD Structure: Up to now, we have neglected the influence of the metallicity and the mass of the progenitor on the structure of the initial WD for a given mass on the main sequence. Such dependencies may become of important if SNe are observed at large distance. On cosmological distance scales, $\mathrm{Z}$ is expected to be correlated with redshift. At the time of the explosion, the WD masses are close to the Chandrasekhar limit. The WD has grown by accretion of $\mathrm{H} / \mathrm{He}$ and subsequent burning from the mass of the central core of a star with less than $\approx 7 M_{\odot}$ (Fig. 6). In the accreted layers, the $\mathrm{C} / \mathrm{O}$ ratio is close to 1 ; however, the initial mass of the $\mathrm{C} / \mathrm{O}$ WD is determined by the stellar evolution. The core mass depends on $M_{M S}$ of the progenitor and on $\mathrm{Z}$.

Here, we want to discuss the size of the metallicity effect using the example of a $7 M_{\odot}$ model with $\mathrm{Z}=0.02$ and 0.004 (Fig.8). Z mainly effects the convection during the stellar Helium burning and, consequently, the size of the $\mathrm{C} / \mathrm{O}$ core and the central $\mathrm{C} / \mathrm{O}$ ratio. We note that the exact size of the effect and its sign depends on mass of the progenitor at the zero age main sequence, and Z. Even for a given mass, the changes are not monotonic, but may change sign from Pop I to Pop II to Pop III $[8,46]$. In addition, the tendency depends sensitively on the assumed physics such as the ${ }^{12} C(\alpha, \gamma){ }^{16} O$-rate (e.g. [43]). Therefore, our example can serve as a guide to estimate the size of this effect.
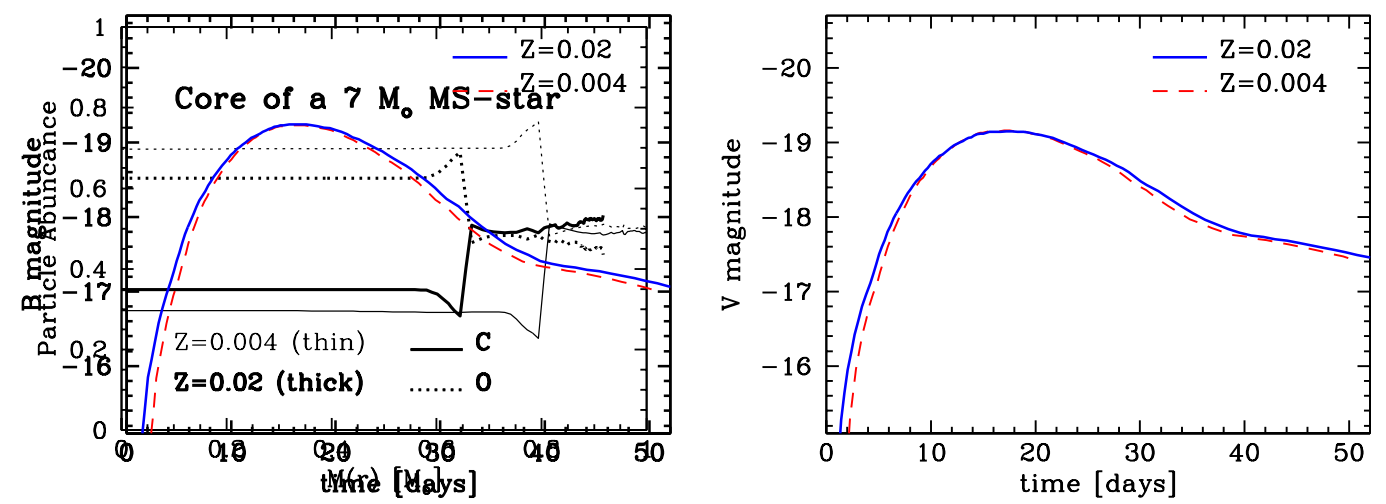

FIGURE 8. Comparison of the final chemical profile of the $\mathrm{C} / \mathrm{O}$ core of a star with $M_{M S}=7 M_{\odot}$ (right) with $\mathrm{Z}=0.02$ and 0.004 , and the corresponding $\mathrm{V}$ light curves (left) of delayed detonation models with identical central density and descriptions for the burning front (see text). 
In agreement with above, the total $\mathrm{C} / \mathrm{O}$ mass determines the explosion energy and, consequently, has the main effect on the light curves. After accretion on the initial core $M_{C}$, the total mass fraction of $M_{C} / M_{C h}$ is 0.75 and 0.61 for the models with $\mathrm{Z}=0.04$ and 0.02 respectively. At the time of the explosion, $\rho_{c}=$ $2.4 \times 10^{9} \mathrm{~g} / \mathrm{cm}^{-3}$. For the burning, $\alpha=0.02$ and $\rho_{t r}=2.410^{7} \mathrm{~g} \mathrm{~cm}^{-3}$ ).

Monochromatic LCs are shown in Fig. 8. As expected from the last section, the main effect on the light curves is caused by the different expansion ratio determined by the integrated $\mathrm{C} / \mathrm{O}$ ratio. The change in the maximum brightness remains small $\left(M_{V}(Z=0.02)-M_{V}(Z=0.04)=-0.02^{m}\right)$ and the rise times are different by about 1 day $\left(t_{V}\left((Z=0.02)=17.4 d\right.\right.$ vs. $\left.t_{V}(Z=0.04)=16.6 d\right)$. The most significant effect is a steeper decline ratio and a reduced ${ }^{56} \mathrm{Ni}$ production for the model with solar Z. This is mainly due to the slower expansion ratio. This translates into a systematic offset of $\approx 0.1^{m}$ in the maximum brightness decline ratio [11]. Using either the streching method or the LCS-method gives similar offsets.

Worth noting is the following trend: For realistic cores, the mean $M_{C} / M_{O}$ tends to be smaller than the canonical value of 1 used in all calculations prior to 1998 (e.g. $[33,50,16])$. Consequently, as a general trend, the rise times are about 1-3 days slower in all models based on the detailed WD structure compared to the models published prior to 1996 ( [16], and references therein).

Acknowledgments: Most of the results reviewed here have been obtained in collaborations with collegues too numerous to be listed all as coauthors. Therefore, I would like to thank here, in particular, A. Chieffi, I. Dominguez, R. Fesen, C.

Gerardy, A. Khokhlov, M. Limongi, E. Müller, K. Nomoto, J. Spyromillo, Y.Stein, O. Straniero, F.K. Thielemann, H.Umeda, L. Wang \& J.C. Wheeler. This work is supported in part by NASA Grant LSTA-98-022.

\section{REFERENCES}

1. Aldering G., Knop R., Nugent P. 2000, AJ, accepted \& astro-ph/0001049

2. Arnett W. D. 1969, Ap. Space Sci., 5, 280

3. Benetti B. 1989, PhD-thesis, University of Padua/Italy

4. Bowers, E.J.C. et al., 1997, MNRAS, 290, 663

5. Branch D., et al. 1998, Phys. Rep., in press

6. Chieffi, A., Limongi, M., and Straniero, O. 1998, ApJ 502, 737

7. Collela P., Woodward P.R. 1984,J.Comp.Phys. 54, 174

8. Dominguez I. Höflich P., Straniero O., Wheeler J.C. 1998, in the Cosmos V, Eds. N. Prantzos \& S. Harissopulos, Editions Frontieres, Paris, p. 248

9. Fisher E. et al. 1998, MNRAS 304, 67

10. Gerardy C.L., Höflich P., Fesen R., Wheeler J.C. 2000, ApJ submitted

11. Hamuy M. et al. 1996,AJ 112, 2438

12. Hansen C.J., Wheeler J.C. 1969, Ap. Space Sci., 3, 464Hansen C.J., Wheeler J.C. 1969, Ap. Space Sci., 3, 464

13. Höflich, P.A, Khokhlov, A.M., Müller, E., 1993, AA, 259, 549

14. Höflich P. 1995, ApJ 443, 89 
15. Höflich P., Khokhlov A., Wheeler J.C. 1995, ApJ 444, 831

16. Höflich P., Khokhlov A. 1996, ApJ 457, 500

17. Höflich P., Khokhlov A., Wheeler J.C., Phillips M., Suntzeff N., Hamuy M., 1996, ApJ 472,81

18. Höflich P., Dominik C., Khokhlov A., Nomoto K., Thielemann K., Wheeler J.C. 1996b, in: Type Ia Supernovae, eds. R. Canal et al., Kluver, p. 659

19. Höflich P., Wheeler J.C., Thielemann F.K 1998, ApJ 495, 617

20. Hoyle F., Fowler W. A. 1960, ApJ 132, 565

21. Iben I.Jr., Tutukov A.V. 1984, ApJS, 54, 335

22. Itoh N., Mitake S., Iyetomi H., Ichimaru S. 1983, ApJ 273, 774

23. Ivanova I.N., Imshennik V.S., Chechetkin V.M. 1974, ApSS, 31, 497

24. Khokhlov A. 1995, ApJ, 449, 695

25. Khokhlov A., Oran E.S., Wheeler J.C. 1997a, ApJ, 478, 678

26. Khokhlov A. 1991ab , A\&A 245, 114 \& L25

27. Müller E., Höflich P. 1994, A\&A 281, 51

28. Niemeyer J.C., Hillebrandt W. 1995, ApJ, 452, 779

29. Nomoto K., Sugimoto S., \& Neo S. 1976, ApSS, 39, L37

30. Nomoto K., Sugimoto D. 1977, PASJ, 29, 765

31. Nomoto K. 1980, IAU-Symp., 93, D. Reidel, p. 295

32. Nomoto K. 1982, ApJ, 253, 798

33. Nomoto K., Thielemann F.-K., Yokoi K. 1984, ApJ 286, 644

34. Nomoto K., Umeda H., Hachisu I., Kato M., Kobayashi C., Tsujimoto T. 2000, in "SNeIa: Theory and Cosmology", eds. J. Truran \& J. Niemeyer, Cambridge University Press

35. Nugent P., et al. 1996, Phys. Rev. Let. 75, 394 \& 1974E

36. Nugent P., Baron E., Branch D., Fisher A., Hausschild P. 1997, ApJ 485, 812

37. Paczyński B.,1985, in: Cataclysmic Variables and Low-Mass X-Ray Binaries, eds. D.Q. Lamb \& J. Patterson, Reidel,Dordrecht, p. 1

38. Phillips M.M. et al. 1987, PASP 90, 592

39. Perlmutter S. et al. 1998, ApJ, in press and astro-ph/9812473

40. Riess A. et al. 1998, AJ 116, 1009

41. Saha A. et al. 1997, ApJ 486, 1

42. Schaller G., Schaerer D., Meynet G., Maeder A. 1992, A\&A Suppl 96, 269

43. Straniero, O., Chieffi, A., and Limongi, M. 1997, ApJ 490, 425

44. Thielemann F.K., Nomoto K., Hashimoto M. 1996, ApJ 460, 408

45. Thielemann F.K., Nomoto K., Yokoi K. 1986, A\&A 158, 17

46. Umeda H., Nomoto K., Yamaoka H., Wanajo S. 1999, ApJ 513, in press

47. Wang L., Höflich P., Wheeler, J.C. 1997, ApJ 487, L29

48. Webbink R.F. 1984, ApJ, 277,355

49. Wheeler, J.C., Höflich, P.A., Harkness R., Spyromillo J. 1998, ApJ, 496, 908

50. Woosley S. E. \& Weaver T. A. 1994, Proc. of Les Houches Session LIV, eds. Bludman et al., North-Holland, 63

51. Woosley S.E., Weaver T.A., 1986,ARAA,24

52. Yamaoka H., Nomoto K., Shigeyama T., Thielemann F., 1992, ApJ, 393, 55 


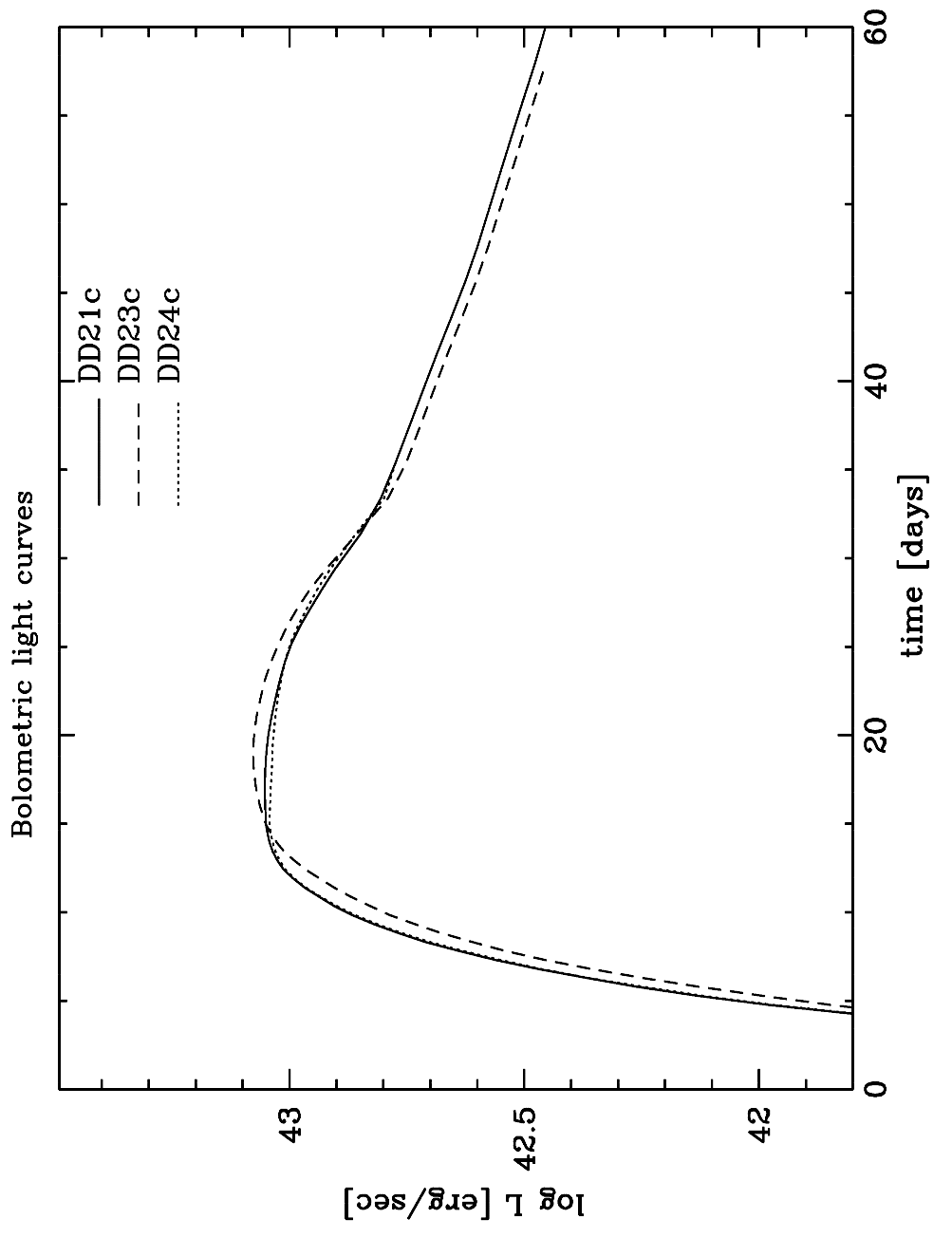



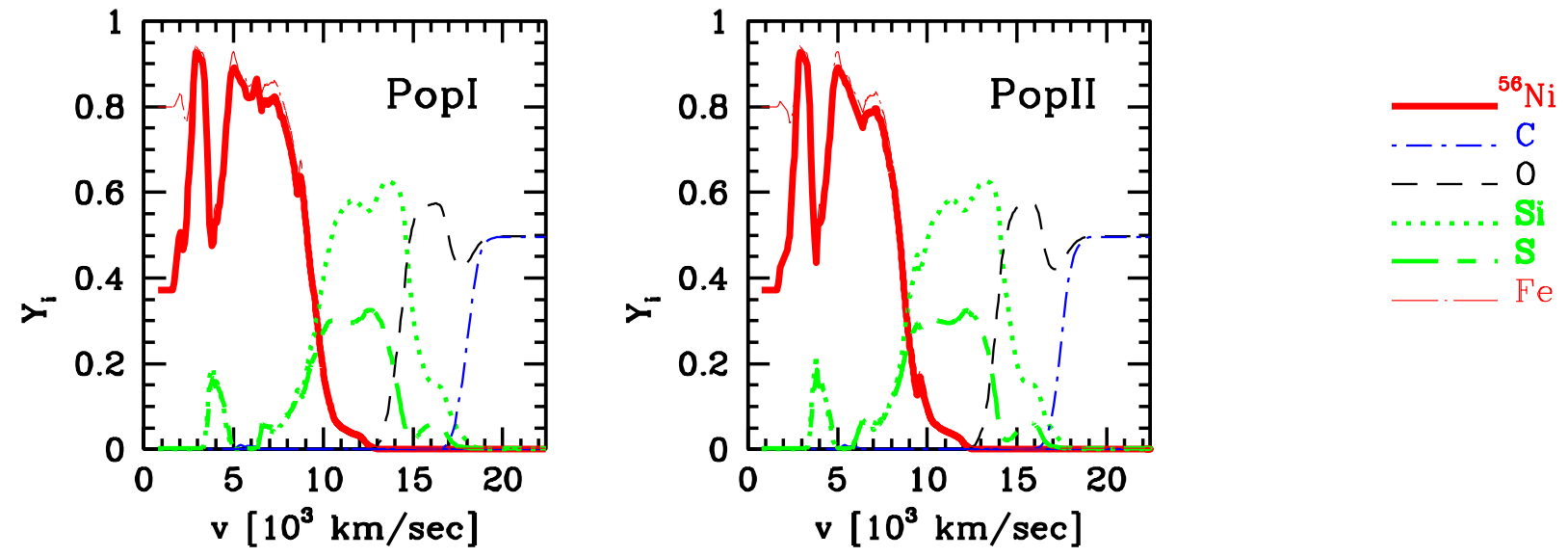